\title{
Charmonium mass in nuclear matter
}

\author{
Su Houng Lee ${ }^{1,2}$ and Che Ming Ko ${ }^{1}$ \\ ${ }^{1}$ Cyclotron Institute and Physics Department, Texas A\&M University, College Station, Texas 77843-3366 \\ ${ }^{2}$ Institute of Physics and Applied Physics and Department of Physics, Yonsei University, Seoul 120-749, Korea
}

(Received 2 August 2002; published 20 March 2003)

\begin{abstract}
The mass shift of charmonium states in nuclear matter is studied in the perturbative QCD approach. The leading-order effect due to the change of gluon condensate in nuclear matter is evaluated using the leadingorder QCD formula, while the higher-twist effect due to the partial restoration of chiral symmetry is estimated using a hadronic model. We find that while the mass of $J / \psi$ in nuclear matter decreases only slightly, those of $\psi(3686)$ and $\psi(3770)$ states are reduced appreciably. Experimental study of the mass shift of charmonium states in nuclear matter can thus provide valuable information on the changes of the QCD vacuum in nuclear medium.
\end{abstract}

Understanding hadron mass changes in nuclear medium and/or at finite temperature can provide valuable information about the QCD vacuum [1-3]. It is also relevant phenomenologically to the interpretation of experimental results from relativistic heavy ion collisions [4], in which a hot dense matter is formed during the collisions. Previous studies have been largely concerned with hadrons made of light quarks [3]. Only recently were there studies of the inmedium masses of hadrons consisting of heavy charm quarks. Using either QCD sum rules $[5,6]$ or the quarkmeson coupling model [7], it has been found that the mass of $D$ meson, which is made of a charm quark and a light quark, is reduced significantly in nuclear medium as a result of the decrease of the light quark condensate. For the $J / \psi$, which consists of a charm and anticharm quark pair, both the QCD sum rules analysis [8] and the LO perturbative QCD calculation $[9,10]$ show that its mass is reduced slightly in the nuclear matter mainly due to the reduction of the gluon condensate in nuclear matter.

The change of hadron masses at finite temperature is best studied using the lattice gauge theory as its prediction is less model dependent. Recent lattice gauge calculations at finite temperature with dynamical quarks have shown that even below critical temperature the interquark potential at large separation approaches an asymptotic value $V_{\infty}(T)$ that decreases with increasing temperature [11]. This transition from a linearly rising interquark potential in free space to a saturated one at finite temperature is due to the decrease in the string tension and the formation of $\bar{Q} q$ and $\bar{q} Q$ pairs, where $q$ denotes a light quark, when the separation of the two heavy quarks $(Q)$ becomes large. The decrease in $V_{\infty}(T)$ can thus be interpreted as a decrease of the open heavy quark meson $(\bar{Q} q$ or $\bar{q} Q)$ mass $m_{H}$, such as the $D$ meson mass, at finite temperature $[12,13]$. Furthermore, the decrease of $m_{H}$ seems to be a consequence of the reduction in the constituent mass of light quark as the temperature dependence of $V_{\infty}(T)$ is similar to that of the chiral condensate $\langle\bar{q} q\rangle$ [14]. This relation between the mass $m_{H}$ and the chiral order parameter also follows naturally from the heavy quark symmetry [15]. With the finite temperature interquark potential, it has been shown via the solution of corresponding Schrödinger equa- tion that the masses of charmonium states are reduced at finite temperature as well $[16,17]$.

At finite density, lattice gauge calculations are at present not feasible for studying the heavy quark potential or the mass $m_{H}$ of the open heavy quark meson. Masses of these heavy quark systems are, however, expected to change appreciably in nuclear medium. Model independent estimates have shown $[18,19]$ that condensates of the lowest dimensional operators $\left\langle\left(\alpha_{s} / \pi\right) G^{2}\right\rangle$ and $\langle\bar{q} q\rangle$ decrease, respectively, by 6 and $30 \%$ at normal nuclear matter, which are significant changes expected only near the critical point at finite temperature [20]. As in the case of finite temperature, the reduction of gluon condensate leads to the softening of the confining part of interquark potential [21], while the decrease of quark condensates implies a drop of the open heavy quark meson mass or $V_{\infty}$ of the heavy quark potential. Both are expected to lead to nontrivial changes in the binding energies of the charmonium states $\psi(3686)$ and $\psi(3770)$, as their wave functions are sensitive to both the confining part and the asymptotic value of the interquark potential.

In this paper, we evaluate the mass shift of $\psi(3686)$ and $\psi(3770)$ due to changes in the gluon and quark condensates in nuclear medium. The effect of the gluon condensate is determined using the leading-order (LO) QCD formula, which was developed in Refs. [9,22] and has been used to study the $J / \psi$ mass in medium [10]. The effect due to the change in quark condensates is difficult to calculate using the quark and gluon degrees of freedom as they appear as higher twist effects in the operator product expansion [23,24]. However, its dominant effect to a heavy quark system is to reduce $V_{\infty}$ as a result of the decrease of $D$ meson in-medium mass, about $50 \mathrm{MeV}$ in normal nuclear matter due to the $30 \%$ reduction in the quark condensate $[5-7,25]$. Therefore, we can study the effect of changing quark condensate on the charmonium states at finite density by using a hadronic model to calculate their mass shifts due to the change of $D$ meson in-medium mass. Combining the effects from changes in the gluon condensate and in $m_{D}$, we find that both $\psi(3686)$ and $\psi(3770)$ masses are reduced appreciably at normal nuclear matter density.

The mass shift of charmonium states in nuclear medium can be evaluated in the perturbative QCD when the charm 
quark mass is large, i.e., $m_{c} \rightarrow \infty$. In this limit, one can perform a systematic operator product expansion (OPE) of the charm quark-antiquark current-current correlation function between the heavy bound states by taking the separation scale $(\mu)$ to be the binding energy of the charmonium $[9,22,26]$. The forward scattering matrix element of the charm quark bound state with a nucleon then has the following form:

$$
T\left(q^{2}=m_{\psi}^{2}\right)=\sum_{n} \frac{C_{n}}{(\mu)^{n}}\left\langle\mathcal{O}_{n}\right\rangle_{N} .
$$

Here, $C_{n}$ is the Wilson coefficient evaluated with the charm quark bound state wave function and $\left\langle\mathcal{O}_{n}\right\rangle_{N}$ is the nucleon expectation value of local operators of dimension $n$.

For heavy quark systems, there are only two independent lowest dimension operators; the gluon condensate $\left[\left\langle\left(\alpha_{s} / \pi\right) G^{2}\right\rangle\right]$ and the condensate of twist-2 gluon operator multiplied by $\alpha_{s}\left[\left\langle\left(\alpha_{s} / \pi\right) G_{\alpha \mu} G_{\nu}^{\alpha}\right\rangle\right]$. These operators can be rewritten in terms of the color electric and magnetic fields $\left\langle\left(\alpha_{s} / \pi\right) E^{2}\right\rangle$ and $\left\langle\left(\alpha_{s} / \pi\right) B^{2}\right\rangle$. Since the Wilson coefficient for $\left\langle\left(\alpha_{s} / \pi\right) B^{2}\right\rangle$ vanishes in the nonrelativistic limit, the only contribution is thus proportional to $\left\langle\left(\alpha_{s} / \pi\right) E^{2}\right\rangle$, similar to the usual second-order Stark effect. We shall thus calculate the mass shift of charmonium states due to change of the gluon condensate in nuclear medium by the QCD secondorder Stark effect [10].

The mass shift of charmonium states to leading order in density is obtained by multiplying the leading term in Eq. (1) by the nuclear density $\rho_{N}$. This gives

$$
\Delta m_{\psi}(\boldsymbol{\epsilon})=-\frac{1}{9} \int d k^{2}\left|\frac{\partial \psi(k)}{\partial \mathbf{k}}\right|^{2} \frac{k}{k^{2} / m_{c}+\boldsymbol{\epsilon}}\left\langle\frac{\alpha_{s}}{\pi} E^{2}\right\rangle_{N} \cdot \frac{\rho_{N}}{2 m_{N}} .
$$

In the above, $m_{N}$ and $\rho_{N}$ are the nucleon mass and the nuclear density, respectively; $\left\langle\left(\alpha_{s} / \pi\right) E^{2}\right\rangle_{N} \sim 0.5 \mathrm{GeV}^{2}$ is the nucleon expectation value of the color electric field and $\epsilon$ $=2 m_{c}-m_{\psi}$. In Ref. [9], the LO mass shift formula was derived in the large charm quark mass limit. As a result, the wave function $\psi(k)$ is Coulombic and the mass shift is expressed in terms of the Bohr radius $a_{0}$ and the binding energy $\epsilon_{0}=2 m_{c}-m_{J / \psi}$. This might be a good approximation for $J / \psi$ but is not realistic for the excited charmonium states as Eq. (2) involves the derivative of the wave function, which measures the dipole size of the system. We have thus rewritten in the above the $\mathrm{LO}$ formula for charmonium mass shift in terms of the QCD parameters $\alpha_{s}=0.84$ and $m_{c}$ $=1.95$, which are fixed by the energy splitting between $J / \psi$ and $\psi(3686)$ in free space [9]. Furthermore, we take wave functions of the charmonium state to be Gaussian with the oscillator constant $\beta$ determined by their squared radii $\left\langle r^{2}\right\rangle$ $=0.47^{2}, 0.96^{2}$, and $1 \mathrm{fm}^{2}$ for $J / \psi, \psi(3686)$, and $\psi(3770)$, respectively, as obtained from the potential models [27]. This gives $\beta=0.52,0.39$, and $0.37 \mathrm{GeV}$ if we assume that these charmonium states are in the $1 S, 2 S$, and $1 D$ states, respectively. Using these parameters, we find that the mass shifts at normal nuclear matter density obtained from the LO QCD formula (2) are $-8,-100$, and $-140 \mathrm{MeV}$ for $J / \psi, \psi(3686)$, and $\psi(3770)$, respectively.

Although the higher twist effects on the charmonium masses are expected to be nontrivial, the result for $J / \psi$ is consistent with those from other nonperturbative QCD studies, such as the QCD sum rules $[8,24]$ and the effective potential model $[28,29]$, which are all based on the dipole interactions between quarks in the charmonium and those in the nuclear matter. To go beyond the leading order in the OPE, we need to calculate the contributions from higher dimensional operators in Eq. (1), which include light quark operators. Explicit calculations from QCD sum rules for $J / \psi$ up to dimension 6 operators [24] show that the effect due to change in the condensates of light quark operators at dimension 6, which include $\langle\bar{q} \Gamma q \bar{q} \Gamma q\rangle$ and $\langle\bar{q} D G q\rangle[23,24]$, is unimportant for the mass shift of $J / \psi$. However, such a calculation cannot be easily generalized to the excited charmonium states $\psi(3686)$ and $\psi(3770)$, where the sum rules do not exist even in the vacuum. On the other hand, the higher twist effects due to the light quark operators can be estimated by considering the coupling of the charmonium to the $\bar{D} D$ states as in the potential model for charmonium states [27]. Therefore, instead of summing up the nonconvergent contributions from the change in the light quark condensates in the OPE of Eq. (1), we estimate its contribution by evaluating the charmed meson one-loop effect on the mass of a charmonium with in-medium $D$ meson mass predicted from the QCD sum rules $[5,6]$ or the quark-meson coupling model [7].

Following the studies in Ref. [30] on $\rho-\pi$ interactions and in Ref. [31] on $\phi-K$ interactions, we use the following Lagrangian for interacting charmonium $\psi$ and $D$ meson:

$$
\mathcal{L}=\frac{1}{2}\left(\left|D_{\mu} \mathbf{D}\right|^{2}-m_{D}^{2}|\mathbf{D}|^{2}\right)-\frac{1}{4} F_{\mu \nu} F^{\mu \nu}+\frac{1}{2} m_{\psi}^{2} \psi_{\mu} \psi^{\mu},
$$

where $F_{\mu \nu}=\partial_{\mu} \psi_{\nu}-\partial_{\nu} \psi_{\mu}$ is the charmonium field strength, $D_{\mu}=\partial_{\mu}-i 2 g_{\psi D D} \psi_{\mu}$, and $\mathbf{D}=\left(D^{0}, D^{+}\right)$.

The coupling constant $g_{\psi D D}$ can be determined using the $3 P 0$ model [32]. In this model, the coupling constant is proportional to the overlap integral between the relative quark wave functions of charmonium and the two outgoing charmed mesons as well as to a coupling parameter $\gamma$, which characterizes the probability of producing a light quarkantiquark pair in the ${ }^{3} P_{0}$ state. The result can be read off from Refs. $[33,34]$ and is given by

$$
g_{\psi D D}^{2}(q)=\gamma^{2} \pi^{3 / 2} \frac{m_{\psi}^{3}}{\beta_{D}^{3}} f_{\psi}\left(q^{2}, r\right) e^{-q^{2} / 2 \beta_{D}^{2}\left(1+2 r^{2}\right)},
$$

where $q$ is the three-momentum of $D$ mesons in the $\psi$ rest frame and $r=\beta / \beta_{D}$ with $\beta_{D}(\beta)$ being the oscillator constant for $D$ meson $(\psi)$ wave function. For the $\beta$ 's, the same values will be used as in the LO QCD calculation. The values for $\gamma$ and $\beta_{D}$ are taken to be 0.35 and $0.31 \mathrm{GeV}$, respectively, to reproduce both the decay width of $\psi(3770)$ to $D \bar{D}$ and the partial decay width of $\psi(4040)$ to $D D, D D^{*}$, and $D^{*} D^{*}[33,35]$. The function $f_{\psi}\left(q^{2}, r\right)$ denotes 


$$
\frac{2^{6} r^{3}\left(1+r^{2}\right)^{2}}{\left(1+2 r^{2}\right)^{5}}
$$

for $J / \psi$,

$$
\begin{aligned}
& \frac{2^{5}\left(3+2 r^{2}\right)^{2}\left(1-3 r^{2}\right)^{2}}{3\left(1+2 r^{2}\right)^{7}} \\
& \quad \times\left(1-\frac{2 r^{2}\left(1+r^{2}\right)}{\left(1+2 r^{2}\right)\left(3+2 r^{2}\right)\left(3 r^{2}-1\right)} \frac{q^{2}}{\beta_{D}^{2}}\right)^{2}
\end{aligned}
$$

for $\psi(3686)$, and

$$
\frac{2^{9} 5 r^{7}}{3\left(1+2 r^{2}\right)^{7}}\left(1-\frac{\left(1+r^{2}\right)}{5\left(1+2 r^{2}\right)} \frac{q^{2}}{\beta_{D}^{2}}\right)^{2}
$$

for $\psi(3770)$.

Because of its momentum dependence, $g_{\psi D D}(q)$ takes into account the form factor at the $\psi D D$ vertex and allows also the coupling of the charmonium to off-shell $D$ mesons. For $\psi(3770)$, it can decay to $D \bar{D}$ in free space, and its onshell coupling constant is $g_{\psi(3770) D D}\left[q=\left(m_{\psi}^{2} / 4-m_{D}^{2}\right)^{1 / 2}\right]$ $=15.4$. The coupling constants at $q=0$ are 15.3, 18.7, and 16.8 for $J / \psi, \psi(3686)$, and $\psi(3770)$, respectively. The value for $J / \psi$ coupling to $D$ mesons is slightly larger than that estimated by the vector meson dominance model [36,37] and by the QCD sum rules [38]. As the $D$ meson momentum increases, its coupling constant to $J / \psi$ has a simple exponential fall off due to the $1 S$ quark wave function of the $J / \psi$. In contrast, the coupling constants of excited charmonium states $\psi(3686)$ and $\psi(3770)$ to $D$ mesons fall off exponentially with the $D$ meson momentum but vanish at certain $q^{2}$ as a result of the nodes in the $2 S$ or $1 D$ wave functions of the excited charmonium states $[33,35]$.

Similar to the method introduced in Ref. [31], we have used the above Lagrangian to evaluate the self-energy of a charmonium due to the $D$ meson loop. After performing the energy integral in the rest frame of the $\psi$, i.e., $k=\left(m_{\psi}, 0\right)$, the invariant part of the polarization $\Pi(k)$ then has the following form:

$$
\begin{aligned}
\Pi(k)= & \frac{1}{6 \pi^{2}} \mathcal{P} \int d q^{2} g_{\psi D D}^{2}\left(q^{2}\right)\left[\frac{q}{\sqrt{m_{D}^{* 2}+q^{2}}}\right. \\
& \left.\times\left(\frac{4 q^{2}}{m_{\psi}^{2}-4 m_{D}^{* 2}-4 q^{2}}+3\right)-\left(m_{D}^{*}=m_{D}\right)\right],
\end{aligned}
$$

where $m_{D}^{*}$ is the in-medium $D$ meson mass and $\mathcal{P}$ denotes that only the principle value of the integral is evaluated. The subtracted term in the above equation is a renormalization constant which is determined by requiring $\Pi\left(k^{2}=m_{\psi}^{2}\right)=0$ when $m_{D}^{*}=m_{D}$. This ensures that the $D$ meson loop does not contribute to the real part of the charmonium self-energy in free space. The mass shift of the charmonium at finite density is then given by $\Delta m_{\psi}=\Pi\left(k^{2}=m_{\psi}^{2}\right)$.

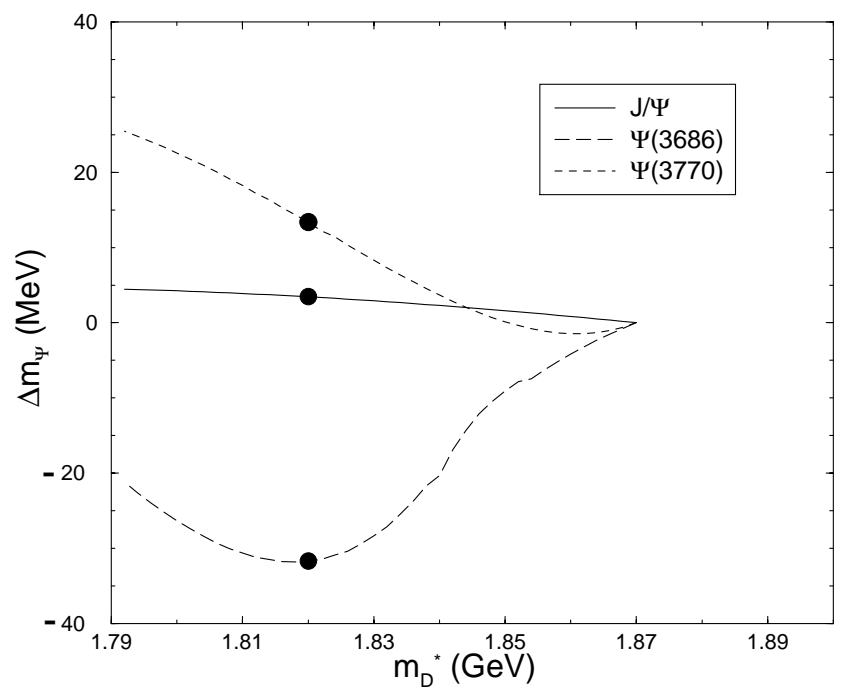

FIG. 1. Mass shifts of charmonium states $J / \psi$ (solid curve), $\psi(3686)$ (long dashed curve), and $\psi(3770)$ (short dashed curve) as functions of $D$ meson in-medium mass $m_{D}^{*}$. The circle indicates the expected mass shifts at normal nuclear matter density.

In Fig. 1, we show the mass shifts of charmonium states as functions of $m_{D}^{*}$. It is seen that the mass shift of $\psi(3770)$ is negative for small negative shift of $D$ meson mass but becomes positive when the $D$ meson mass drop is large. In contrast, the mass shift of $\psi(3686)$ is negative for all negative mass shifts of the $D$ meson. This difference can be understood from Eq. (5), where the integral is a convolution of the form factor $g_{\psi D D}^{2}\left(q^{2}\right)$ with the terms in the square bracket, which are singular when $q^{2}=m_{\psi}^{2} / 4-m_{D}^{* 2}$ and $q^{2}$ $=m_{\psi}^{2} / 4-m_{D}^{2}$. The integrand thus changes signs whenever the $D$ meson momentum $q$ passes through these singularities and finally becomes negative when $q^{2}$ is larger than any of the singularities, which correspond to the energies of the virtual intermediate $D$ meson states. As in second-order perturbation theory, the contribution is attractive when the energy of the intermediate state is larger than the charmonium mass. However, the form factor decreases exponentially with $q^{2}$ and can even be zero, the large negative contribution expected for a constant form factor is suppressed, leading thus to an increase of the $\psi(3770)$ mass when $m_{D}-m_{D}^{*} \geqslant 10$ $\mathrm{MeV}$. On the other hand, the singularity of the integrand in Eq. (5) in the case of $\psi(3683)$ occurs only when $2 m_{D}^{*}$ falls below its mass and therefore has only a small positive contribution when $q^{2}$ is very small, leading to a reduction of its mass for any $D$ meson mass shift. For $J / \psi$, we find that its mass only increases slightly with dropping $D$ meson mass and depends weakly on $m_{D}^{*}$. For $m_{D}-m_{D}^{*}=50 \mathrm{MeV}$, which is the expected mass shift of $D$ meson at normal nuclear matter density, the mass shift of $J / \psi$ is about $3 \mathrm{MeV}$. This result is consistent with that from the QCD sum rules [24] and is also expected from the potential model [27], where the $J / \psi$ wave function has only a small $D \bar{D}$ component. We note that the density dependence of the mass shifts of charmonium states, particularly the excited ones, is nonlinear if we use a linearly density-dependent $D$ in-medium meson $m_{D}^{*}$ $=m_{D}-50 \rho / \rho_{0} \mathrm{MeV}$ in the denominator of Eq. (5). On the 
other hand, the mass shift obtained from the LO QCD formula in Eq. (2) depends linearly on the nuclear density.

Adding the mass shift from the $D$ meson loop effect to the result from the LO QCD calculation, we find that masses of charmonium states are changed by the following amount at normal nuclear matter density:

$$
\begin{gathered}
\Delta m_{J / \psi}=-8+3 \mathrm{MeV}, \\
\Delta m_{\psi(3686)}=-100-30 \mathrm{MeV}, \\
\Delta m_{\psi(3770)}=-140+15 \mathrm{MeV},
\end{gathered}
$$

where the first number represents the shift from the LO QCD while the second number is from the $D$ meson loop. The above results thus show that masses of excited charmonium states are reduced significantly in nuclear matter, largely due to the nontrivial decrease of the in-medium gluon condensate in the LO QCD formula for their masses.

The mass shifts of both $\psi(3686)$ and $\psi(3770)$ in nuclear medium are large enough to be observed in experiments involving $\bar{p}-A$ annihilation as proposed in the future accelera- tor facility at the German Heavy Ion Accelerator Center (GSI) [39]. In these experiments, $\psi(3770)$ and $\psi(3686)$ produced inside a heavy nucleus will be studied via the dilepton spectrum emitted from their decays. While the lifetime of $J / \psi$ has been shown to remain constant in nuclear matter, those of $\psi(3686)$ and $\psi(3770)$ are reduced to less than $5 \mathrm{fm} / c$ due to increases in their width in nuclear matter [33]. Therefore, an appereciable fraction of produced excited charmonium states in these experiments are expected to decay inside the nucleus [40], leading to an observable shift of the peak positions in the dilepton spectrum. The observation of such shifts in the masses of excited charmonium states in these experiments would give us valuable information on the nontrivial changes of the QCD vacuum in nuclear medium and on the origin of masses in QCD.

This paper is based on work supported by the National Science Foundation under Grant No. PHY-0098805 and the Welch Foundation under Grant No. A-1358. S.H.L was also supported by the Brain Korea 21 project of Korean Ministry of Education and by KOSEF under Grant No. 1999-2-111005-5.
[1] G.E. Brown and M. Rho, Phys. Rev. Lett. 66, 2720 (1991).

[2] T. Hatsuda and K. Kunihiro, Phys. Rep. 247, 221 (1994).

[3] S.H. Lee, Nucl. Phys. A638, 183c (1998).

[4] C.M. Ko and G.Q. Li, J. Phys. G 221, 673 (1996); C.M. Ko, V. Koch, and G.Q. Li, Annu. Rev. Nucl. Part. Sci. 47, 505 (1997).

[5] A. Hayashigaki, Phys. Lett. B 487, 96 (2000).

[6] P. Morath, W. Weise, and S.H. Lee, Proceedings of 17th Lisbon Autumn School on Perturbative and Nonperturbative QCD (World Scientific, Singapore, 1999), p. 425.

[7] K. Tsushima et al., Phys. Rev. C 59, 2824 (1999).

[8] F. Klingl et al., Phys. Rev. Lett. 82, 3396 (1999).

[9] M.E. Peskin, Nucl. Phys. B156, 365 (1979).

[10] M.E. Luke et al., Phys. Lett. B 288, 355 (1992).

[11] F. Karsch et al., Nucl. Phys. A605, 579 (2001).

[12] S. Digal et al., Phys. Lett. B 514, 57 (2001).

[13] H. Satz, hep-ph/0111265.

[14] A. Peikert et al., Nucl. Phys. A73, 468c (1999).

[15] N. Isgur and M.B. Wise, Phys. Lett. B 232, 113 (1989); 237, 527 (1990).

[16] C.Y. Wong, Phys. Rev. C 65, 034902 (2002); nucl-th/0112064; C.Y. Wong et al., nucl-th/0112023.

[17] T. Hashimoto et al., Phys. Rev. Lett. 57, 2123 (1986).

[18] E.G. Drukarev and E.M. Levin, Prog. Part. Nucl. Phys. 27, 77 (1991)

[19] T. Hatsuda and S.H. Lee, Phys. Rev. C 46, R34 (1992).

[20] S.H. Lee, Phys. Rev. D 40, 2484 (1989).
[21] M.A. Shifman, Nucl. Phys. B173, 13 (1980); H.G. Dosch and Y.A. Simonov, Phys. Lett. B 205, 339 (1988).

[22] G. Bhanot and M.E. Peskin, Nucl. Phys. B156, 391 (1979).

[23] S.N. Nikolaev et al., Nucl. Phys. B213, 285 (1983).

[24] S. Kim and S.H. Lee, Nucl. Phys. A679, 517 (2001).

[25] G.Q. Li and C.M Ko, Phys. Lett. B 338, 118 (1994).

[26] Y. Oh, S. Kim, and S.H. Lee, Phys. Rev. C 65, 067901 (2002).

[27] E. Eichten et al., Phys. Rev. D 17, 3090 (1978); 21, 203 (1980).

[28] S.J. Brodsky et al., Phys. Rev. Lett. 64, 1011 (1990).

[29] D.A. Wasson, Phys. Rev. Lett. 67, 2237 (1991).

[30] C. Gale and J. Kapusta, Phys. Rev. D 43, 3080 (1991).

[31] C.M. Ko et al., Phys. Rev. C 45, 1400 (1992).

[32] A. Le Yaouanc et al., Phys. Rev. D 8, 2223 (1973); 9, 1415 (1974); 11, 1272 (1975).

[33] B. Friman, S.H. Lee, and T. Song, Phys. Lett. B 548, 153 (2002).

[34] T. Barnes et al., Phys. Rev. D 55, 4157 (1997).

[35] A. Le Yaouanc et al., Phys. Lett. 71B, 397 (1977).

[36] S.G. Matinyan and B. Müller, Phys. Rev. C 58, 2994 (1998).

[37] Z.W. Lin and C.M. Ko, Phys. Rev. C 62, 034903 (2000); W. Liu, C.M. Ko, and Z.W. Lin, ibid. 65, 015203 (2002).

[38] R.D. Matheus et al., Phys. Lett. B 541, 265 (2002).

[39] See http://www.gsi.de/GSI-future.

[40] Y.S. Golubeva et al., nucl-th/0212074. 